A C G publications organic communications

\title{
Synthesis, spectral characterization, antimicrobial activity and docking studies against DNA Gyrase-A of new 4-chloro-3- nitrobenzene sulfonamide derivatives
}

\section{K. Pushpa Kumar ${ }^{1,2}$, Reddi Mohan Naidu Kalla ${ }^{3 *}$, \\ Mavallur Varalakshmi $\odot^{4}$, Chintha Venkataramaiah ${ }^{5 *} \oplus$,K. Swetha Kumari $\odot^{6}$, Jayakumar Kannali $\oplus^{7}$, D. Venkatramana Reddy $\oplus^{2,8}$ and Charmathi Nagaraju*(i) 1}

\author{
${ }^{1}$ Department of Chemistry, S. V. University, Tirupati-517501, Andhra Pradesh, India., Italia \\ ${ }^{2}$ Department of Chemistry, S. G. Govt. Degree \& PG College, Pileru, Sri Venkateswara University, \\ Tirupati-517502, A.P., India \\ ${ }^{3}$ Department of Science and Humanities, Sri Venkateswara Engineering College, Tirupati-517507, \\ Andhra Pradesh, India \\ ${ }^{4}$ Center for Applied Sciences, Sree Vidyanikethan Engineering College, A. Rangampet, Tirupati, 517502, \\ Andhra Pradesh, India \\ ${ }^{5}$ Department of Zoology, Sri Venkateswara Vedic University, Tirupati-517502, Andhra Pradesh, India \\ ${ }^{6}$ Department of Biosciences and Sericulture, Sri Padmavati Mahila Visvavidyalayam, Tirupati-517 502, \\ Andhra Pradesh, India \\ ${ }^{7}$ Department of Chemistry, S. V. University, Tirupati-517502, Andhra Pradesh, India \\ ${ }^{8}$ Department of Chemistry, S. G. Government Degree College, Piler, India
}

(Received November 02, 2020; Revised December 21, 2020; Accepted December 22, 2020)

\begin{abstract}
A series of new 4-chloro-3-nitrobenzene sulfonamide derivatives 3(a-j) were synthesized from 4-chloro-3nitrobenzene sulfonyl chloride by reacting various amino acid esters and amines $\mathbf{2}(\mathbf{a}-\mathbf{j})$ in high yields. The structures of all the synthesized compounds were characterized by the IR, NMR $\left({ }^{1} \mathrm{H} \&{ }^{13} \mathrm{C}\right)$, mass and elemental analyses. Further, all the synthesized compounds were tested for the antimicrobial activity and docking studies were carried out with DNA Gyrase-A. Most of the compounds showed good to moderate antimicrobial activities and binding affinity towards DNA Gyrase-A structure.
\end{abstract}

Keywords: 4-Chloro-3-nitrobenzene; amino acid esters; antimicrobial activity; docking studies; DNA Gyrase A; sulfonamides.@ 2020 ACG Publications. All rights reserved.

\section{Introduction}

Nowadays, heterocyclic motifs are found everywhere in pharmaceutical and agrochemical industries. The literature reports revealed that majority of the organic heterocycles are with sulfur and nitrogen containing compounds. The nitrogen and sulfur framework is one of the most privileged sources

\footnotetext{
*Corresponding authors: E-Mail: reddinaidu.svu@ gmail.com (R.M.N Kalla) ; rajuchamarthi10@ gmail.com (C.Nagaraju); chintharamana9@gmail.com (C. Venkataramaiah)
} 
for developing the new antimicrobial agents ${ }^{1}$. Among these, the sulfonamides have been extensively deliberated for their chemotherapeutic test. Their significant role as anti-malarial, antibacterial, and antileprotic medium is well renowned ${ }^{2,3}$. Recently, particular sulfonamide derivatives having fascinating antibacterial activity have been widely studied by Quantitive Structure-activity Relation-ship \& Molecular Modeling (QSAR) method ${ }^{4}$. Antimicrobial treatment for infections with $S$. aureus often includes sulfonamides which were used to reduce nosocomial infections ${ }^{5}$. Even though the sulfonamide therapy has been reduced, due to development of more efficient antimicrobial targets and the ongoing increase in the resistance of bacterial species, sulfonamides have become salient alternative option in order to heal methicillin resistant S. aureus (MRSA) Staphylococci infections.

Furthermore, sulfonamides are efficient active groups with extensive function in organic materials, agriculture, and dyes ${ }^{6}$. They are commonly used in veterinary drugs for medicinal purposes to battle bacterial diseases ${ }^{7}$. Sulfonamide compounds signify a class of derivatives having fascinating pharmacological properties. Numerous legislatures of this class of compounds were described to have HIV protease inhibitors ${ }^{8,9}$, carbonic anhydrase inhibitors ${ }^{1,10}$, antiepileptic agents ${ }^{11,12}$, anticonvulsant agents ${ }^{13}$ and are used as ETA, being a selective antagonist ${ }^{14,15}$. In addition, sulfonamides have become important class of pharmacophoric parts and constitute more than 200 drugs in the market. The fragments bearing these functionalities show auspicious roles in current drug discovery and medicinal chemistry. Also, several sulfonamide derivatives were found to exhibit abundant biological activities like antitumor ${ }^{16}$, hypoglycemic ${ }^{17}$, antithyroid ${ }^{18}$, anticarbonic anhydrase ${ }^{19}$, anti-inflammatory ${ }^{20,21}$, diuretic ${ }^{22}$, antimicrobial ${ }^{23}$, COX inhibition and anti-impotence activities ${ }^{24}$.

DNA gyrase $\mathrm{e}^{25,26}$ is an essential bacterial enzyme that catalyze the ATP-dependent negative supercoiling of double-stranded closed-circular DNA. Gyrase belongs to the class of enzymes namely topoisomerases that are involved in the control of topological transitions of DNA. The mechanism by which gyrase is able to influence the topological state of DNA molecules is of inherent interest from an enzymological standpoint. In addition, much attention has been focused on DNA gyrase as an intracellular target to a number of antibacterial agents and as a paradigm for other DNA topoisomerases ${ }^{27}$.

Led by the above facts, we herein report the synthesis of new 4-chloro-3-nitrobenzene sulfonamide derivatives and evaluated their antimicrobial activity, also carried out molecular docking studies against DNA gyrase enzyme.

\section{Experimental}

\subsection{Chemical Material and Apparatus}

All the chemicals were obtained from Sigma-Aldrich and Merck and were used without further purification. The solvents used for spectroscopic studies and other physical studies were of reagent grade and were purified by literature methods. The melting points were determined on Ez-Melt Melting Point Apparatus equipped with Digital Imaging Processing Technology of Standard Research Systems using open capillary method and are uncorrected. The IR spectra were recorded on Bruker Alpha-Eco ATR-FTIR (Attenuated Total Reflection-Fourier Transform Infrared) Interferometer with single reflection sampling module equipped with $\mathrm{ZnSe}$ crystal and were reported in reciprocal centimeters $\left(\mathrm{cm}^{-1}\right) .{ }^{1} \mathrm{H}$ and ${ }^{13} \mathrm{C}$ NMR spectra were recorded in $\mathrm{CDCl}_{3}$ solvent on Bruker FT-NMR $400 \mathrm{MHz}$ as well as Jeol FT - NMR $400 \mathrm{MHz}$ spectrometers operating at $400 \mathrm{MHz}$ for ${ }^{1} \mathrm{H}$ NMR and $100 \mathrm{MHz}$ for ${ }^{13} \mathrm{C}$ NMR. The $1 \mathrm{H}$ and $13 \mathrm{C}$ NMR chemical shifts were referenced to tetramethyl silane and reported in delta, $\delta$ values in ppm. Multiplicities were abbreviated as s (singlet), d (doublet), t (triplet), q (quartet), dd (double doublet), $\mathrm{m}$ (multiplet) and brs (broad singlet). The mass spectra were recorded on LCMS-2010A Shimadzu Mass Spectrometer equipped with electron spray ionization (ESI) source at atmospheric pressure in positive ion mode. A 10$5 \mathrm{M}$ solution of $3 \mathrm{~g}$ and $3 \mathrm{j}$ were prepared in HPLC grade DMSO separately. Each solution was infused into the mass spectrometer @ $6 \mu \mathrm{L} / \mathrm{min}$. The optimized conditions set for ESI were as shown: dry $\mathrm{N}_{2}$ flow rate $12 \mathrm{~L} / \mathrm{min}$ at $200{ }^{\circ} \mathrm{C}$; the nebulizer $\mathrm{N}_{2}$ was operated at $35 \mathrm{psi}$; capillary voltage was 400 Volts; the skimmer was at $65 \mathrm{~V}$; and the fragmenter was maintained at a voltage of $90 \mathrm{~V}$; quantification was performed in selected ion monitoring (SIM) mode using target ions $\mathrm{m} / \mathrm{z} 325.0$ for $3 \mathrm{j}$ and $\mathrm{m} / \mathrm{z} 350.20$ for $3 \mathrm{k}$. The CHN analysis was carried out on Flash EA 1112 Thermo Finnigan for $3 \mathrm{~g}$ and $3 \mathrm{j}$ compounds and found consistent with literature values. 


\subsection{Biological Materials and Apparatus}

Tris borate EDTA (TBA), ethidium bromide, 7-aminoactinomycine D (7-AAD), 4', 6-diammidino2-fenilindolo (DAPI), propidio iodine, annexine, $\mathrm{CHO}$ (Chinese Hamster Ovary) and Hela (by Henrietta Lacks, whose cancer cells of the uterine cervix were the first to be immortalized) cell lines were purchased by Thermofischer Scientific, while JetPEI from PolyPlus. KMS-12-BM and LP1 cell lines were taken from a patient suffering from multiple myeloma (MM). The factors necessary to cell growth [Dulbecco's Minimal Essential Medium (DMEM), fetal bovine serum (FBS), amino acids, sodium pyruvate, antibiotics, Reduced Serum Medium (OptiMEM), trypsin], $p$ DNA and siRNA were purchased from Invitrogen ${ }^{\mathrm{TM}}$ (Thermofischer Scientific). Electrophoresis was performed in Agarose Gels, 4\% TBA (Agarose 4g in 100 $\mathrm{mL}$ TBA buffer) and Agarose was purchased from Sigma-Aldrich. Cytofluorometric analysis was performed on Beckman Coulter's Gallios 10/3 Cytofluorimeter. Fluorescence emissions were evaluated and quantified by a double fluorescence FM YG100 microscope produced by Suzhou Flyingman Precision Instruments Co. Ltd.

\subsection{Chemistry}

2.3.1. Procedure for the synthesis of (S)-methyl 2-(4-chloro-3-nitrophenylsulfonamido)-3-(4hydroxyphenyl)propanoate $(\mathbf{3 g})$

To the stirred solution of 4-Chloro-3-nitrobenzene sulfonyl chloride (1) $(0.001 \mathrm{~mol}, 0.256 \mathrm{~g})$ dissolved in THF $(10 \mathrm{~mL})$, methyl-L-tyrosinate $(0.001 \mathrm{~mol}, 0.195 \mathrm{~g})$ was added with constant stirring at $20^{\circ} \mathrm{C}$ in the presence of triethyl amine (TEA) and the reaction mixture was refluxed at $40^{\circ} \mathrm{C}$ for $2 \mathrm{~h}$. The progress of the reaction was monitored by TLC. After the completion of reaction, the reaction mixture was concentrated under rota evaporator. The crude reaction mixture was purified by column chromatography using ethyl acetate and hexane (3:7) as mobile phase to obtain (S)-methyl 2-(4-chloro-3-nitrophenylsulfonamido)-3(4-hydroxyphenyl) propanoate (3g) as a pure product. The same experimental procedure was adopted for the synthesis of the remaining target compounds $\mathbf{3}(\mathbf{a}-\mathbf{f})$ and $\mathbf{3}(\mathbf{h}-\mathbf{j})$ using different substituted amino acid esters and amines.

\subsubsection{Spectral Data for Synthesized Compounds}

Methyl 2-(4-chloro-3-nitrophenylsulfonamido)-3-methylbutanoate (3a): White solid, yield: 90\%, mp: 163 ${ }^{\circ} \mathrm{C}$; IR $\left(\mathrm{cm}^{-1}\right): 3451(-\mathrm{OH}), 3254(-\mathrm{NH}), 1437\left(\mathrm{Ar}^{\left.-\mathrm{NO}_{2}\right)}\right), 1358$ and $1187\left(\mathrm{SO}_{2}\right.$ Asym and Sym), $954(\mathrm{~S}-\mathrm{N}$ Sym); ${ }^{1} \mathrm{H}$ NMR (400 MHz, $\left.\mathrm{CDCl}_{3}\right): \delta 8.62(\mathrm{~d}, J=8.0 \mathrm{~Hz}, 1 \mathrm{H}), 8.24(\mathrm{dd}, 1 \mathrm{H}), 7.98(\mathrm{~d}, 1 \mathrm{H}), 7.75$ (brs, $\left.1 \mathrm{H}\right)$, $5.40(\mathrm{~s}, 1 \mathrm{H}), 3.76(\mathrm{~m}, 1 \mathrm{H}), 3.73\left(\mathrm{~s}, 3 \mathrm{H},-\mathrm{OCH}_{3}\right), 3.10(\mathrm{~d}, J=6.5 \mathrm{~Hz}, 3 \mathrm{H}) ; 2.90(\mathrm{~d}, J=6.5 \mathrm{~Hz}, 3 \mathrm{H}) ;{ }^{13} \mathrm{C} \mathrm{NMR}$ $\left(100 \mathrm{MHz}, \mathrm{CDCl}_{3}\right): \delta 171.5,155.6,148.0,138.6,134.9,131.1,130.3,129.9,129.3,124.6,115.9,58.3$, 52.0, 35.8. MW 398;ESI-MS: $416\left[\mathrm{M}+\mathrm{H}_{2} \mathrm{O}\right]$.

Methyl 2-(4-chloro-3-nitrophenylsulfonamido)-3-(1H-indol-3-yl)propanoate (3b): White solid, yield: 88\%, mp: $155^{\circ} \mathrm{C}$; IR $\left(\mathrm{cm}^{-1}\right)$ : $3264(-\mathrm{NH}), 1733\left(\mathrm{CO}_{2} \mathrm{Et}\right), 1440\left(\mathrm{Ar}-\mathrm{NO}_{2}\right), 1332$ and $1168\left(\mathrm{SO}_{2}\right.$ Asym and Sym), 908 (S-N Sym); ${ }^{1} \mathrm{H}$ NMR (400 MHz, $\left.\mathrm{CDCl}_{3}\right): 8.62$ (d, $\left.J=8.0 \mathrm{~Hz}, 1 \mathrm{H}\right), 8.24$ (dd, 1H), 7.98 (d, 1H), 7.70 (brs, 1H), 7.65-7.06 (m, 4H, Ar-H), 3.86-3.76 (m, 1H), $3.73(\mathrm{~s}, 3 \mathrm{H}), 3.47-3.17(\mathrm{~m}, 2 \mathrm{H}) ;{ }^{13} \mathrm{C}$ NMR $(100$ $\left.\mathrm{MHz}, \mathrm{CDCl}_{3}\right): \delta 171.7,147.8,138.6,136.6,134.7,131.1,130.1,127.5,124.6,123.1,121.6,119.9,59.4$, 51.9, 29.7. MW 398; ESI-MS: $416\left[\mathrm{M}+\mathrm{H}_{2} \mathrm{O}\right]$.

Ethyl 2-(4-chloro-3-nitrophenylsulfonamido)-3-hydroxypropanoate (3c): Brown solid, yield: 89\%, mp: $160{ }^{\circ} \mathrm{C}$; IR $\left(\mathrm{cm}^{-1}\right)$ : $3260(-\mathrm{NH}), 1733\left(\mathrm{CO}_{2} \mathrm{Me}\right), 1436\left(\mathrm{Ar}-\mathrm{NO}_{2}\right), 1352$ and $1186\left(\mathrm{SO}_{2}\right.$ Asym and Sym $), 949$ (S-N Sym); ${ }^{1} \mathrm{H}$ NMR $\left(400 \mathrm{MHz}, \mathrm{CDCl}_{3}\right): \delta 8.62(\mathrm{~d}, J=8.0 \mathrm{~Hz}, 1 \mathrm{H}), 8.24$ (dd, 1H), 7.98 (d, H), 7.68 (brs, $1 \mathrm{H}), 4.42-4.16(\mathrm{~m}, 4 \mathrm{H}), 4.01(\mathrm{~m}, 1 \mathrm{H}), 3.70(\mathrm{~s},-\mathrm{OH}, 1 \mathrm{H}), 1.25(\mathrm{t}, J=6.2 \mathrm{~Hz}, 3 \mathrm{H}) ;{ }^{13} \mathrm{C} \mathrm{NMR}(100 \mathrm{MHz}$, $\left.\mathrm{CDCl}_{3}\right): \delta 171.6,147.8,138.8,134.7,131.1,129.9,124.6,61.4,60.0,59.4,14.2$.

Ethyl 2-(4-chloro-3-nitrophenylsulfonamido)acetate (3d): White solid, yield: $92 \%$, mp: $147{ }^{\circ} \mathrm{C}$; IR (cm1): $3254(-\mathrm{NH}), 1736\left(\mathrm{CO}_{2} \mathrm{Me}\right), 1432\left(\mathrm{Ar}_{-} \mathrm{NO}_{2}\right), 1356$ \& $1182\left(\mathrm{SO}_{2}\right.$ Asym and Sym), $942(\mathrm{~S}-\mathrm{N} \mathrm{Sym}) ;{ }^{1} \mathrm{H}$ 
NMR (400 MHz, $\left.\mathrm{CDCl}_{3}\right): \delta 8.62(\mathrm{~d}, J=8.0 \mathrm{~Hz}, 1 \mathrm{H}), 8.24(\mathrm{dd}, 1 \mathrm{H}), 7.98(\mathrm{~d}, 1 \mathrm{H}), 7.69$ (brs, $\left.1 \mathrm{H}\right), 4.08-3.96$ $(\mathrm{m}, 2 \mathrm{H}), 3.86-3.75(\mathrm{~m}, 2 \mathrm{H}), 1.24(\mathrm{t}, J=6.5 \mathrm{~Hz}, 3 \mathrm{H}) ;{ }^{13} \mathrm{C} \mathrm{NMR}\left(100 \mathrm{MHz}, \mathrm{CDCl}_{3}\right): \delta 169.6,149.8,138.8$, $134.7,131.1,129.9,124.6,60.9,44.3,14.2$.

4-chloro-N-((1S)-2-(3,4-difluorophenyl)cyclopropyl)-3-nitrobenzenesulfonamide (3e): Yellow solid, yield: 88\%, mp: $170{ }^{\circ} \mathrm{C}$; IR (cm $\left.{ }^{-1}\right)$ : $3251(-\mathrm{NH}), 1436\left(\mathrm{Ar}^{-\mathrm{NO}_{2}}\right), 1353$ and $1180\left(\mathrm{SO}_{2}\right.$ Asym and Sym), $956(\mathrm{~S}-\mathrm{N}$ Sym); ${ }^{1} \mathrm{H}$ NMR (400 MHz, $\left.\mathrm{CDCl}_{3}\right): \delta 8.62(\mathrm{~d}, J=8.0 \mathrm{~Hz}, 1 \mathrm{H}), 8.24(\mathrm{dd}, 1 \mathrm{H}), 7.98(\mathrm{~d}, 1 \mathrm{H}), 7.73$ (brs, $\left.1 \mathrm{H}\right)$, $7.19(\mathrm{~d}, J=6.5 \mathrm{~Hz}, 1 \mathrm{H}), 7.10(\mathrm{~d}, J=8.0 \mathrm{~Hz}, 1 \mathrm{H}), 6.78(\mathrm{~d}, 4.5 \mathrm{~Hz}, 1 \mathrm{H}), 1.70-1.68(\mathrm{~m}, 2 \mathrm{H}), 0.70-0.55(\mathrm{~m}$, $2 \mathrm{H}) ;{ }^{13} \mathrm{C}$ NMR (100 MHz, CDCl3): $\delta 149.0,147.8,146.0,140.4,138.8,134.7,131.1,129.9,124.6,123.7$, 116.6, 113.7, 28.6, 22.1, 13.5.

1-((4-chloro-3-nitrophenyl)sulfonyl)indoline (3f): Brown solid, yield: $87 \%$, mp: $150{ }^{\circ} \mathrm{C}$; IR $\left(\mathrm{cm}^{-1}\right)$ : 1435(Ar-NO $\left.{ }_{2}\right), 1352\left(\mathrm{SO}_{2}\right.$ Asym), $945(\mathrm{~S}-\mathrm{N} \mathrm{Sym}) ;{ }^{1} \mathrm{H}$ NMR $\left(400 \mathrm{MHz}, \mathrm{CDCl}_{3}\right)$ : and $8.62(\mathrm{~d}, J=8.0 \mathrm{~Hz}$, $1 \mathrm{H}), 8.24(\mathrm{dd}, 1 \mathrm{H}), 7.98(\mathrm{~d}, 1 \mathrm{H}), 7.02-6.55(\mathrm{~m}, 4 \mathrm{H}), 3.54(\mathrm{t}, J=4.0 \mathrm{~Hz}, 2 \mathrm{H}), 3.01(\mathrm{t}, J=4.0 \mathrm{~Hz}, 2 \mathrm{H}) ;{ }^{13} \mathrm{C}$ NMR (100 MHz, $\left.\mathrm{CDCl}_{3}\right): \delta 147.8,143.0,138.6,134.9,130.1,129.9,127.6,126.8,125.3,124.6,120.5$, 113.5, 42.9, 26.6.

(S)-methyl 2-(4-chloro-3-nitrophenylsulfonamido)-3-(4-hydroxyphenyl)propanoate $(3 \mathrm{~g}): \mathrm{IR}\left(\mathrm{cm}^{-1}\right)$ : 3256 (-NH), $1436\left(\mathrm{Ar}_{-} \mathrm{NO}_{2}\right), 1352$ \& $1165\left(\mathrm{SO}_{2}\right.$ Asym and Sym), 963 (S-N Sym); ${ }^{1} \mathrm{H} \mathrm{NMR}\left(400 \mathrm{MHz}, \mathrm{CDCl}_{3}\right)$ : $\delta 8.08(\mathrm{~s}, 1 \mathrm{H}), 7.73(\mathrm{dd}, 2 \mathrm{H}), 7.75-7.08(\mathrm{~m}, 5 \mathrm{H}), 5.24(\mathrm{brs}, 1 \mathrm{H}), 4.24(\mathrm{~d}, J=4.8 \mathrm{~Hz}, 1 \mathrm{H}), 3.66(\mathrm{~s}, 3 \mathrm{H}), 3.12-$ $2.92(\mathrm{~m}, 2 \mathrm{H}) \mathrm{ppm} ;{ }^{13} \mathrm{C} \mathrm{NMR}\left(100 \mathrm{MHz}, \mathrm{CDCl}_{3}\right): \delta 171.1,134.7,132.8,130.9,129.3,128.8,127.5,124.3$, 57.2, 52.9, 39.2 ppm; ESI-MS; 416 [M+2].

1-((4-chloro-3-nitrophenyl)sulfonyl)-4-(4-chloro-phenyl)Piperazine (3h): Yellow solid, yield: 93\%, mp: $161{ }^{\circ} \mathrm{C}$; IR $\left(\mathrm{cm}^{-1}\right): 1450\left(\mathrm{Ar}-\mathrm{NO}_{2}\right), 1340 \& 1132\left(\mathrm{SO}_{2}\right.$ Asym and Sym), 906 (S-N Sym); ${ }^{1} \mathrm{H}$ NMR (400 $\left.\mathrm{MHz}, \mathrm{CDCl}_{3}\right): \delta 8.62(\mathrm{~d}, J=8.0 \mathrm{~Hz}, 1 \mathrm{H}), 8.24(\mathrm{dd}, 1 \mathrm{H}), 7.98(\mathrm{~d}, J=8.0 \mathrm{~Hz}, 1 \mathrm{H}), 7.36(\mathrm{dd}, 2 \mathrm{H}), 6.65(\mathrm{dd}$, 2H), $3.24(\mathrm{~d}, 4 \mathrm{H}) ;{ }^{13} \mathrm{C} \mathrm{NMR}\left(100 \mathrm{MHz}, \mathrm{CDCl}_{3}\right): \delta 148.0,147.8,134.7,133.6,130.9,130.1,129.6,127.3$, $124.4,115.8,53.0,48.5$.

1-((4-chloro-3-nitrophenyl)sulfonyl)-4-(4-nitrophenyl)piperazine (3i): Yellow solid, yield: 92\%, mp: 171 ${ }^{\circ} \mathrm{C}$; IR $\left(\mathrm{cm}^{-1}\right): 454\left(\mathrm{Ar}-\mathrm{NO}_{2}\right), 1348$ \& $1139\left(\mathrm{SO}_{2}\right.$ Asym and Sym), 910 (S-N Sym); ${ }^{1} \mathrm{H}$ NMR $(400 \mathrm{MHz}$, $\left.\mathrm{CDCl}_{3}\right): \delta 8.62(\mathrm{~d}, J=8.0 \mathrm{~Hz}, 1 \mathrm{H}), 8.24(\mathrm{dd}, 1 \mathrm{H}), 8.13(\mathrm{~d}, 1 \mathrm{H}), 7.98(\mathrm{~d}, 2 \mathrm{H}), 7.20(\mathrm{~d}, 2 \mathrm{H}), 3.24(\mathrm{~d}, 4 \mathrm{H}),{ }^{13} \mathrm{C}$ NMR (100 MHz, $\left.\mathrm{CDCl}_{3}\right): \delta$ 155.8, 147.8, 137.3, 134.9, 133.4, 131.1, 130.1, 129.9, 124.7, 124.5, 112.2, $53.2,48.3$.

4-chloro- $N$-(2,3-dihydro-1H-inden-1-yl)-3-nitrobenzenesulfonamide (3j): White solid, yield: 90\%, mp: 158 ${ }^{\circ} \mathrm{C}$; IR $\left(\mathrm{cm}^{-1}\right): 3252(-\mathrm{NH}), 1428\left(\mathrm{Ar}^{-N}{ }_{2}\right), 1356$ \& $1191\left(\mathrm{SO}_{2}\right.$ Asym and Sym), 958 (S-N Sym); ${ }^{1} \mathrm{H}$ NMR $\left(400 \mathrm{MHz}, \mathrm{CDCl}_{3}\right): \delta 8.60(\mathrm{~d}, 1 \mathrm{H}), 8.17(\mathrm{dd}, 1 \mathrm{H}), 7.98(\mathrm{~d}, 1 \mathrm{H}), 7.73($ brs, $1 \mathrm{H}), 7.22-7.12(\mathrm{~m}, 4 \mathrm{H}), 4.01(\mathrm{t}$, $J=3.5,1 \mathrm{H}), 2.92(\mathrm{~m}, 1 \mathrm{H}), 2.84(\mathrm{~m}, 1 \mathrm{H}), 2.32(\mathrm{~m}, 1 \mathrm{H}), 2.18(\mathrm{~m}, 1 \mathrm{H}) ;{ }^{13} \mathrm{C}$ NMR $\left(100 \mathrm{MHz}, \mathrm{CDCl}_{3}\right): \delta 148.0$, 143.3, 140.0, 138.6, 134.9, 131.1, 129.9, 126.2, 124.5, 124.4, 58.3, 33.6, 28.9.

\subsection{Biological Assay}

\subsubsection{Antibacterial Activity}

All the newly synthesized compounds were evaluated for their in vitro antibacterial activity against the growth of four bacterial strains such as Pseudomonas aeruginosa, Klebsiella pneumoniae, Escherichia coli, and Staphylococcus aureus by using agar well diffusion method ${ }^{28}$. Chloramphenicol was used as a standard. The zones of inhibition are summarized in Table S1. The detailed procedure has been provided in the supporting information. 


\subsubsection{Antifungal Activity}

All the title compounds were tested for their in vitro antifungal activity against the fungal strains Trichoderma viride, Aspergillus niger, Aspergillus flavus, Penicillium chrysogenum by using poison plate method $^{29}$. Nystatin was used as a standard. The zones of inhibition are summarized in Table S2. The detailed procedure has been provided in the supporting information.

\subsubsection{Molecular Docking Studies}

Molecular docking studies of the newly synthesized compounds 3(a-j) was carried out against DNA Gyrase A protein. Streptomycin and Norfloxacin was used as a reference drug using the docking module implemented in Pyrx 2010.12. The results of the binding were presented in Table 1.

\section{Results and Discussion}

\subsection{Chemistry} Figure 1.

The newly synthesized 4-chloro-3-nitrobenzene sulfonamide derivatives $\mathbf{3}(\mathbf{a}-\mathbf{j})$ were represented in

The structures of all the newly synthesized compounds were characterized by IR, NMR $\left({ }^{1} \mathrm{H},{ }^{13} \mathrm{C}\right)$, mass and elemental analysis. The details are provided in the experimental section and supporting information. In the IR spectrum of compound 3g, the absorption appeared in the region 3451, 3254, 1437 $\mathrm{cm}^{-1}$ corresponds to the functional groups of $-\mathrm{OH},-\mathrm{NH}$ and $\mathrm{Ar}-\mathrm{NO}_{2}$ stretching frequencies respectively. In ${ }^{1} \mathrm{HNMR}$ spectrum, the chemical shift resonated in the region of $\delta 7.75 \mathrm{ppm}$ appeared as broad singlet and it corresponds to $-\mathrm{NH}$ protons. The chemical shift at the region of $\delta 3.73 \mathrm{ppm}$ appeared as a singlet and is assigned to methyl protons. The chemical shifts at the regions of $\delta 5.40-8.62 \mathrm{ppm}$ corresponds to the aromatic protons. In ${ }^{13} \mathrm{CNMR}$ spectrum, chemical shift at the region $\delta 171.5 \mathrm{ppm}$ corresponds to carbonyl carbon and at $\delta 148.0 \mathrm{ppm}$ corresponds to cyclic nitrogen adjacent to aromatic carbon. Further, the structure of compound $\mathbf{3 g}$ was confirmed by the mass and the elemental analyses.

\subsection{Biological Assay}

\subsubsection{Antibacterial Activity}

All the newly synthesized compounds were tested for their antibacterial activity against bacterial strains Pseudomonas aeruginosa, Klebsiella pneumoniae, Escherichia coli, Staphylococcus aureus at 100 $\mu \mathrm{g} / \mathrm{mL}$ concentration by using agar well disc diffusion method. Chloramphenicol was used as a standard. Among all the tested compounds, compound $\mathbf{3 d}, \mathbf{3 e}$ and $\mathbf{3 f}$ showed effective inhibition when compared with other compounds and this might be due to the presence of tertiary sulfonamide and fluoro substituted amines against all the bacterial strains. Compound 3f showed effective inhibition against all the bacterial strains. This might be due to the presence of electron withdrawing nitro group. The zone of inhibition of all the title compounds were presented in Supporting Information Table S1 and Figure S1.

\subsubsection{Antifungal Activity}

Antifungal activity of the newly synthesized compounds $\mathbf{3}(\mathbf{a}-\mathbf{j})$ were tested against the fungal strains such as Trichoderma viridae, Aspergillus niger, Aspergillus flavus, Penicillium chrysogenum by using poison plate method at $100 \mu \mathrm{g} / \mathrm{mL}$. Nystatin was used as a standard drug. Compound $\mathbf{3 f}$ have shown potent antifungal activity against all the tested fungal strains due to the presence of nitro group at meta position. The zones of inhibition of all the title compounds were presented in Table S2 (see supporting information). 


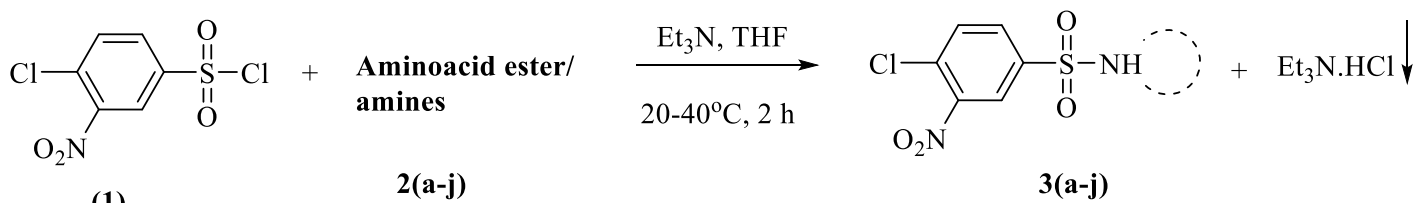

(1)

2(a-j)<smiles>COC(=O)C(NS(=O)(=O)c1ccc(Cl)c([N+](=O)[O-])c1)C(C)C</smiles>

(3a)<smiles>CCOC(=O)CNS(=O)(=O)c1ccc(Cl)c([N+](=O)[O-])c1</smiles>

(3d)<smiles>COC(=O)[C@H](Cc1ccc(O)cc1)NS(=O)(=O)c1ccc(Cl)c([N+](=O)[O-])c1</smiles>

(3g)<smiles>O=[N+]([O-])c1cc(S(=O)(=O)NC2CCc3ccccc32)ccc1Cl</smiles>

(3j)<smiles>COC(=O)C(Cc1ccccc1)NS(=O)(=O)c1ccc(Cl)c([N+](=O)[O-])c1</smiles>

(3b)<smiles>O=[N+]([O-])c1cc(S(=O)(=O)NC2CC2c2ccc(F)c(F)c2)ccc1Cl</smiles>

(3e)<smiles>O=[N+]([O-])c1cc(S(=O)(=O)N2CCN(c3ccc(Cl)cc3)CC2)ccc1Cl</smiles><smiles>CCOC(=O)C(CO)NS(=O)(=O)c1ccc(Cl)c([N+](=O)[O-])c1</smiles>

(3c)<smiles>O=[N+]([O-])c1cc(S(=O)(=O)N2CCc3ccccc32)ccc1Cl</smiles>

(3f)

Figure 1. Synthesis of 4-chloro-3-nitrobenzene sulfonamide derivatives 3(a-j)

\subsubsection{Molecular Docking Analysis}

In order to provide strength to the synthesized compounds $\mathbf{3}(\mathbf{a}-\mathbf{j})$ molecular docking studies was carried out against DNA Gyrase A protein. Streptomycin, Norfloxacin was used as a reference drug using the docking module implemented in Pyrx 2010.12. 
Table 1. H-bonds, binding affinities and energy profiles of compounds 3(a-j)

Compound B E Binding interaction Bond Length $\left(\mathrm{A}^{\mathrm{o}}\right) \quad$ Bond Angle $\left(^{\circ}\right) \quad$ Bond Type

\begin{tabular}{|c|c|c|c|c|c|}
\hline Streptomycin & -6.9 & $\begin{array}{l}\text { Arg } 139 \text { CG...HN } \\
\text { Leu } 135 \mathrm{CD} \ldots \mathrm{HN} \\
\text { His } 132 \mathrm{CB} \ldots \mathrm{OH} \\
\text { Asp } 53 \mathrm{CG} \ldots . \mathrm{OC} \\
\text { Asp } 53 \mathrm{OC} \ldots \mathrm{OC} \\
\text { Asp } 58 \mathrm{OD} \ldots \mathrm{OH} \\
\text { Asp } 58 \mathrm{OD} \ldots \mathrm{HN} \\
\text { His } 132 \mathrm{ND} \ldots \mathrm{OC} \\
\text { His } 132 \mathrm{ND} \ldots \mathrm{OC} \\
\text { His } 132 \mathrm{OC} \ldots \mathrm{OH}\end{array}$ & $\begin{array}{l}2.2 \\
2.7 \\
2.5 \\
3.4 \\
2.9 \\
2.0 \\
2.5 \\
2.8 \\
2.7 \\
2.5\end{array}$ & $\begin{array}{l}124.4 \\
125.7 \\
125.0 \\
116.7 \\
118.9 \\
118.6 \\
116.4 \\
126.2 \\
120.0 \\
119.8\end{array}$ & $\begin{array}{l}\text { H- don } \\
\text { H- don } \\
\text { H- acc } \\
\text { H- acc } \\
\text { H- acc } \\
\text { H- acc } \\
\text { H- don } \\
\text { H- acc } \\
\text { H- acc } \\
\text { H- acc }\end{array}$ \\
\hline Norfloxacin & -7.3 & Leu 264 CA...OC & 2.0 & 114.7 & $\mathrm{H}-\mathrm{acc}$ \\
\hline $3 a$ & -7.7 & $\begin{array}{l}\text { Pro } 218 \mathrm{CA} \ldots . . \mathrm{HO} \\
\text { Pro } 218 \mathrm{CA} \ldots . . \mathrm{HO} \\
\text { Leu } 264 \mathrm{CA} . . . \mathrm{OH} \\
\text { Leu } 264 \mathrm{CA} . . . \mathrm{OC} \\
\text { Thr } 219 \mathrm{CB} \ldots . \mathrm{HO} \\
\text { Asp } 297 \mathrm{CA} \ldots . . \mathrm{HN} \\
\text { Met } 301 \mathrm{CA} . . . \mathrm{OS}\end{array}$ & $\begin{array}{l}2.1 \\
2.3 \\
1.9 \\
2.4 \\
2.3 \\
2.1 \\
2.1\end{array}$ & \begin{tabular}{c|}
123.9 \\
112.1 \\
114.7 \\
93.1 \\
102.9 \\
122.4 \\
115.2 \\
\end{tabular} & $\begin{array}{l}\text { H- don } \\
\text { H- don } \\
\text { H- don } \\
\text { H- acc } \\
\text { H- don } \\
\text { H- don } \\
\text { H- acc }\end{array}$ \\
\hline $3 \mathbf{b}$ & -7.0 & $\begin{array}{l}\text { Asn } 165 \text { ND...ON } \\
\text { Asn } 165 \text { CG...HO } \\
\text { Thr } 171 \text { CA...HN } \\
\text { Thr } 171 \text { CA...OC }\end{array}$ & $\begin{array}{l}2.2 \\
2.8 \\
2.3 \\
2.7\end{array}$ & $\begin{array}{l}116.5 \\
141.0 \\
114.6 \\
117.5\end{array}$ & $\begin{array}{l}\text { H- acc } \\
\text { H- don } \\
\text { H- don } \\
\text { H- acc }\end{array}$ \\
\hline $3 c$ & -6.2 & $\begin{array}{l}\text { Asp } 297 \text { OC...HO } \\
\text { Leu } 264 \mathrm{HN} . . . \mathrm{OH} \\
\text { Thr } 219 \text { CB....HO }\end{array}$ & $\begin{array}{l}2.1 \\
2.0 \\
2.5\end{array}$ & $\begin{array}{l}122.4 \\
114.7 \\
119.9\end{array}$ & $\begin{array}{l}\text { H- don } \\
\text { H- acc } \\
\text { H- don }\end{array}$ \\
\hline 3d & -6.4 & $\begin{array}{c}\text { Asn } 269 \text { ND...OS } \\
\text { Gln } 267 \text { CA...OH } \\
\text { Arg } 91 \text { CZ...ON }\end{array}$ & $\begin{array}{l}2.3 \\
2.6 \\
2.2\end{array}$ & $\begin{array}{l}120.3 \\
123.9 \\
121.8\end{array}$ & $\begin{array}{l}\mathrm{H}-\mathrm{acc} \\
\mathrm{H}-\mathrm{acc} \\
\mathrm{H}-\mathrm{acc}\end{array}$ \\
\hline $3 \mathbf{e}$ & -7.5 & $\begin{array}{l}\text { Gly } 110 \text { NH....ON } \\
\text { Phe } 109 \text { CG...ON } \\
\text { Asn } 108 \text { CG...HO } \\
\text { Gly } 107 \text { OC...HO } \\
\text { Asp } 297 \text { CA....NH }\end{array}$ & $\begin{array}{l}2.7 \\
2.2 \\
2.4 \\
2.1 \\
2.2\end{array}$ & $\begin{array}{c}113.4 \\
99.5 \\
121.5 \\
121.6 \\
122.4\end{array}$ & $\begin{array}{l}\text { H- acc } \\
\text { H- acc } \\
\text { H- don } \\
\text { H- don } \\
\text { H- acc }\end{array}$ \\
\hline $3 f$ & -7.4 & $\begin{array}{l}\text { Gly } 267 \text { CG...ON } \\
\text { Asn } 269 \text { ND...OS } \\
\text { Arg } 91 \mathrm{CZ} \ldots . . \mathrm{ON}\end{array}$ & $\begin{array}{l}2.6 \\
2.3 \\
2.2 \\
\end{array}$ & $\begin{array}{l}114.9 \\
120.3 \\
121.8\end{array}$ & $\begin{array}{l}\mathrm{H}-\mathrm{acc} \\
\mathrm{H}-\mathrm{acc} \\
\mathrm{H}-\mathrm{acc}\end{array}$ \\
\hline $3 g$ & -6.8 & $\begin{array}{l}\text { Pro } 218 \text { OC...HO } \\
\text { Thr } 219 \text { CA...HN } \\
\text { Leu } 264 \text { HC...OC } \\
\text { Arg } 518 \text { CZ....ON }\end{array}$ & $\begin{array}{l}2.3 \\
2.6 \\
1.9 \\
2.5\end{array}$ & $\begin{array}{l}123.9 \\
119.2 \\
114.7 \\
122.4\end{array}$ & $\begin{array}{l}\text { H- don } \\
\text { H- don } \\
\text { H- acc } \\
\text { H- acc }\end{array}$ \\
\hline $3 h$ & -7.7 & $\begin{array}{l}\text { Asp } 115 \text { CG...HO } \\
\text { Asp } 115 \text { CG....HO }\end{array}$ & $\begin{array}{l}2.6 \\
1.9\end{array}$ & $\begin{array}{l}117.7 \\
127.0\end{array}$ & $\begin{array}{l}\text { H- don } \\
\text { H- don }\end{array}$ \\
\hline $3 \mathbf{i}$ & -8.3 & $\begin{array}{c}\text { Gln } 267 \text { CG....ON } \\
\text { Ala } 117 \text { CA....ON } \\
\text { Asn } 269 \text { ND....OS } \\
\text { Asp } 87 \text { CG....HO } \\
\text { Arg } 91 \text { CZ.....ON }\end{array}$ & $\begin{array}{l}2.5 \\
2.2 \\
2.4 \\
2.4 \\
2.3\end{array}$ & $\begin{array}{l}114.3 \\
114.9 \\
120.3 \\
125.4 \\
121.8\end{array}$ & $\begin{array}{l}\text { H- acc } \\
\text { H- acc } \\
\text { H- acc } \\
\text { H- don } \\
\text { H- acc }\end{array}$ \\
\hline $\mathbf{3 j}$ & -7.5 & Val 112 CB....OS & 2.6 & 110.8 & $\mathrm{H}-\mathrm{acc}$ \\
\hline
\end{tabular}

The docking results of DNA Gyrase A showed that compounds $\mathbf{3 a}, \mathbf{3 e}, \mathbf{3 h}, \mathbf{3 i}, \mathbf{3} \mathbf{j}$, have significant binding modes, with dock scores of $-8.3,-7.7,-7.7,-7.5,-7.5$, when compared with the control drug Streptomycin (-6.9) and Norfloxacin (-7.3) respectively. The H-bonds, binding affinities and energy profiles of 
compounds $\mathbf{3}(\mathbf{a}-\mathbf{j})$ along with reference drugs, towards the active site amino acids of the enzyme are summarized in Table 1 . The binding modes of compounds $\mathbf{3 a}, \mathbf{3 e}, \mathbf{3 h}, \mathbf{3 i}, \mathbf{3 j}$, suggested that they fitted more stably into the DNA Gyrase binding pocket. Hence, the present investigation demonstrate that the synthesised compounds will be the promising next generation anti-microbial drugs, which can be effectively used in the treatment of microbial and other related infections.

\section{Conclusion}

We have synthesized a series of new 4-chloro-3-nitrobenzene sulfonamide derivatives $\mathbf{3}(\mathbf{a}-\mathbf{j})$ in an easy method with high yields. All the newly synthesized compounds were evaluated for their in vitro and in silico antimicrobial activity to understand the biological potency and binding capacities with the protein, DNA gyrase A. The results revealed that most of the compounds showed good antimicrobial activity against all the microbial strains and will stand as the core molecules for the discovery of potential antimicrobial drug candidates in future.

\section{Acknowledgements}

The authors are grateful to the Department of Chemistry and Zoology, Sri Venkateswara University, Tirupati for providing lab facilities. The co-author, Reddi Mohan Naidu Kalla, is thankful to the management and principal of S V Engineering College at Tirupati for their support and encouragement during the research.

\section{Supporting Information}

Supporting information accompanies this paper on http://www.acgpubs.org/journal/organiccommunications

\section{ORCID}

K. Pushpa Kumar: 0000-0002-4042-3396

Reddi Mohan Naidu Kalla: 0000-0001-7689-2242

Mavallur Varalakshmi: 0000-0001-6638-6813

Chintha Venkataramaiah: 0000-0002-0493-6047

K.Swetha Kumari: 0000-0002-8316-1891

Jayakumar Kannali: 0000-0002-8004-5967

D. Venkatramana Reddy: 0000-0003-1701-7231

Chamarthi Nagaraju: 0000-0001-6566-2118

\section{References}

[1] Gokcen, T.; Al, M.; Topal, M.; Gulcin, I.; Ozturk, T.; Goren, A.C. Synthesis of some natural sulphonamide derivatives as carbonic anhydrase inhibitors. Org. Commun. 2017, 10, 15-23.

[2] Benedetti, P.G.D. Advances in drug research. Volume 16. Edited by: Testa B. Academic Press. London and New York; 1987:227.

[3] Mengelers, M. J.; Hougee, P. E.; Jansson, L. H.; Van Miert, A.S. Structure-activity relationship between antibacterial activities and physicochemical properties of sulfonamides. J. Vet. Pharmacol. Therap. 1997, 20, 276-283.

[4] Akçam, F. Z.; Karaslan, D.; Doğan, M.; Yaylı, G. Microbiological surveillance in the Intensive Care Unit: A tertiary hospital experience. Med. Sci. Monit. 2006, 12, 81-85.

[5] Monika G.; Mohan, C. Development of drugs based on imidazole and benzimidazole bioactive heterocycles: recent advances and future directions. Med. Chem. Res. 2016, 25, 173-210. 
[6] Gumus, M.; Sert, Y.; Koca, I. Synthesis, characterization and theoretical studies of novel sulfonamidealdehydes derivatives having tautomeric forms. Org. Commun. 2019, 12, 176-187.

[7] Yotphan, S.; Sumunnee, L.; Beukeaw, D.; Buathongjan, C.; Reutrakul, V. Iodine-catalyzed expeditious synthesis of sulfonamides from sulfonyl hydrazides and amines. Org. Biomol. Chem. 2016, 14, 590-597.

[8] Tan, G. T.; Wickramasingle, A.; Verma, S.; Singh, R.; Hughes, S. H.; Pezzuto, J. M.; Baba, M.; Mohan, P. Potential anti-AIDS naphthalenesulfonic acid derivatives. synthesis and inhibition of HIV-1 induced cytopathogenesis and HIV-1 and HIV-2 reverse transcriptase activities. J. Med. Chem. 1992, 35, 4846-4853.

[9] Mohan, P.; Singh, R.; Baba, M. Potential anti-AIDS agents. Synthesis and antiviral activity of naphthalenesulfonic acid derivatives against HIV-1 and HIV-2. J. Med. Chem. 1991, 34, 212-217.

[10] Boriack, P. A.; Christianson, D.W.; Kingery-Wood, J.; Whitesides, G.M. Secondary Interactions Significantly Removed from the Sulfonamide Binding Pocket of Carbonic Anhydrase II Influence Inhibitor Binding Constants. J. Med. Chem. 1995, 38, 2286-2291.

[11] Leppik, I. E. Antiepileptic Drugs in Development: Prospects for the Near Future. Epilepsia 1994, 35, S29-S40.

[12] Tanimukai, H.; lnui, M,; Hariguchi, S.; Kaneko, Z. Antiepileptic property of inhibitors of carbonic anhydrase. Biochem. Pharmacol. 1995, 14, 961-970.

[13] Flaherty, P. T.; Greenwood, T. D.; Manheim, A. L.; Wolfe, J. F. Synthesis and evaluation of N(phenylacetyl)trifluoromethanesulfonamides as anticonvulsant agents. J. Med. Chem. 1996, 39, 1509-1513.

[14] Stein, P. D.; Floyd, D. M.; Bisaha, S.; Dickey, J.; Girotra, R. N.; Gougoutas, J. Z.; Kozlowsk, M.; Lee, V. G.; Liu, E. C. K.; Malley, M. F.; McMullen, D.; Metchell, C.; Moteland, S.; Murugesan, N.; Serafino, R.; Webb, M. L.; Zhang, R.; Hunt, J. T. Discovery and structure-activity relationships of sulfonamide ETA-selective antagonists. J. Med. Chem. 1995, 38, 1344-1354.

[15] Closel, M.; Breu, V.; Bum, K.; Cassal, J. M.; Fischli, W. Gray, G. A.; Hirth, G.; Loffier, B. N.; Muller, M.; Neidhart, W.; Ramuz, H. Pathophysiological role of endothelin revealed by the first orally active endothelin receptor antagonist. Nature, 1993, 365, 759-761.

[16] Eldehna, W. M.; Nocentini, A.; Al-Rashood, S.T., Hassan, G.S; Alkahtani, H. M.; Almehizia, A. A.; Reda, A. M.; Abdel-Aziz, H. A.; Supuran, C.T. Tumor-associated carbonic anhydrase isoform IX and XII inhibitory properties of certain isatin-bearing sulfonamides endowed with in vitro antitumor activity towards colon cancer. Bioorg. Chem. 2018, 81, 425-432.

[17] Thornber, C. W. Isosterism and molecular modification in drug design. Chem. Soc. Rev. 1979, 8, 563-580.

[18] Ogden, R. C.; Flexner, C. W. Protease inhibitors in AIDS therapy (Marcel Dekker; Inc, New York, 2001, pp. 101-118.

[19] Nishimori, I.; Vullo, D.; Innocenti, A.; Scozzafava, A.; Mastrolorenz, A.; Supuran, C. T. Carbonic anhydrase inhibitors: Inhibition of the transmembrane isozyme XIV with sulfonamides. Bioorg. Med. Chem. Lett. 2005, $15,3828-3833$.

[20] Li, J. J.; Anderson, D.; Burton, E. G.; Cogburn, J. N.; Collins, J. T.; Garland, D. J.; Gregory, S. A.; Huang, H. C.; Isakson, P. C.; Koboldt, C. M.; Logusch, E. W.; Norton, M. B.; Perkins, W. E.; Reinhard, E. J.; Seibert, K.; Veenhuizem, A. W.; Zang, Y.; Reitz, D. B. Diarylspiro[2.4]heptenes as orally active, highly selective cyclooxygenase-2 inhibitors: synthesis and structure-activity relationships. J. Med. Chem. 1996, 39, $253-266$.

[21] Rao, D. S.; Madhava, G.; Raju, C. N.; Balaji, M.; Madhusudhana, S.; Rani, A. U. 2-Amino-2,3-dihydro-1H-

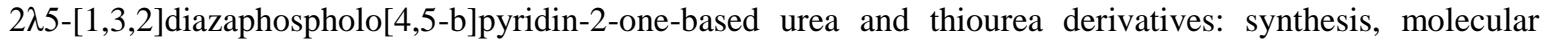
docking study and evaluation of anti-inflammatory and antimicrobial activities. Med. Chem. Res. 2016, 25, 751-768.

[22] Boyd, A. E. Sulfonylurea receptors, ion channels, and fruit flies. Diabetes 1988, 37, 847-850.

[23] Narayana, K.V.; Rao, D.S.; Madhava, G.; Venkateswarlu, N.; Vijaya, T.; Raju, C. N. Sulfonamide and carbamate derivatives of 6-chloropurine:synthesis, characterization and antimicrobial activity evaluation. $\mathrm{Org}$. Commun. 2016, 9, 42-53.

[24] Claudiu, T. S.; Angela, C.; Andrea, S. Protease inhibitors of the sulfonamide type: Anticancer, antiinflammatory, and antiviral agents. Med. Res. Rev. 2003, 23, 535-558.

[25] Nitiss, J. L. Yeast as a genetic model system for studying topoisomerase inhibitors. Adv. Pharmacol. 1994, 29, 201-226.

[26] Maxwell, A.; Gellert, M. Mechanistic aspects of DNA topoisomerases. Adv. Protein Chem. 1986, 38, 69-107.

[27] Brino, L.; Urzhumtsev, A.; Mousli, M.; Bronner, C.; Mitschler, A.; Oudet, P.; Moras, D. Dimerization of Escherichia coli DNA-gyrase B provides a structural mechanism for activating the ATPase catalytic center. $J$. Biol. Chem. 2000, 275, 9468-9475. 
[28] Tian, M.Y.; Zhao, X.G.; Wu, X.H.; Hong, Y.; Chen, Q.; Liu, X.L.; Zhou, Y. Chemical composition, antibacterial and cytotoxic activities of the essential oil from Ficus tikoua Bur. Rec. Nat. Prod. 2020, 14, 219224.

[29] Sen Utsukarci, B.; Gurdal, B.; Bilgin, M.; Satana, D .; Demirci, B.; Tan, N.; Mat, A. Biological activities of various extracts from Salvia cassia Sam. ex Rech.f. and chemical composition of Its most active extract. Rec. Nat. Prod. 2019, 13, 24-36.

[30] Bagamboula, C. F.; Uyttendaele, M.; Debevere, J. Inhibitory effect of thyme and basil essential oils, carvacrol, thymol, estragol, linalool and p-cymene towards Shigella sonnei and S. flexneri. Food Microbiol. 2004, $21,33-$ 42.

[31] Ustuner, T.; Kordali, S.; Bozhuyuk, A.U.; Kesdek, M. Investigation of pesticidal activities of essential oil of Eucalyptus camaldulensis Dehnh. Rec. Nat. Prod. 2018, 12, 557-568.

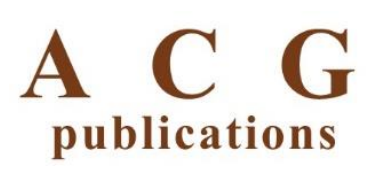

(C) 2020ACG Publications 